\title{
New insights into the study of magnetic field in the clumpy torus of AGN using near-infrared polarimetry
}

\author{
E. Lopez-Rodriguez ${ }^{1,2}$, C. Packham ${ }^{1,2}$, S. Young ${ }^{3}$, M. Elitzur ${ }^{4}$, N. A. Levenson ${ }^{5}$, R. E. Mason ${ }^{6}$, C. Ramos Almeida ${ }^{7,8}$, \\ A. Alonso-Herrero ${ }^{*}$, T. J. Jones ${ }^{10}$, and E. Perlman ${ }^{11}$

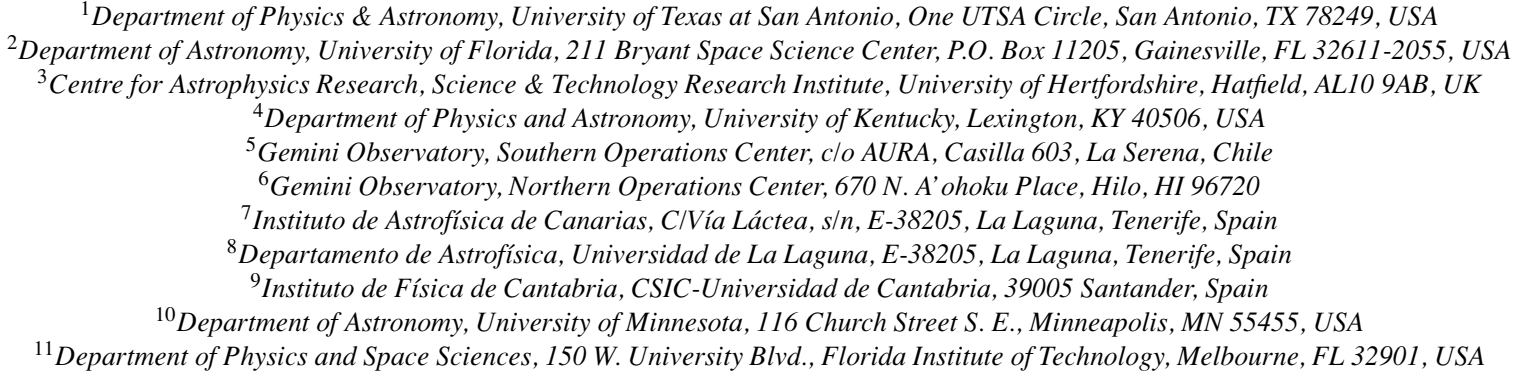

(Received November 14, 2012; Revised April 10, 2013; Accepted May 20, 2013; Online published October 24, 2013)

We present $\mathrm{J}, \mathrm{H}$ and $\mathrm{K}_{\mathrm{n}}$ imaging polarimetry of IC5063. NIR polarimetry observations may advance our understanding of the mechanisms of polarisation and hence magnetic field strength in the torus of AGN. In these proceedings we summarize the polarisation results of the central $1.2^{\prime \prime}$ aperture $(\sim 263 \mathrm{pc})$ of IC5063. We present a simple polarising model to account for various mechanisms of polarisation in the central regions of IC5063. The model is consistent with dichroic absorption from diffuse stellar emission through dust in the nuclear bulge and electron scattering as dominant mechanisms of polarisation at $\mathbf{J}$ and $\mathrm{H}$. Dichroic absorption from aligned dust grains within the torus of IC5063 is dominant at $K_{n}$ with a visual extinction of $A_{v}=48 \pm 2$ mag by the torus. Through the use of various components to the central engine of IC5063, we estimated the intrinsic polarisation from dichroic absorption to be $\mathrm{P}_{\mathrm{K}_{\mathrm{n}}}^{\text {dic }}=12.5 \pm 2.7 \%$. The dust grain alignment mechanism is assumed to be produced by paramagnetic relaxation. In this case, the intrinsic polarisation, $\mathrm{P}_{\mathrm{K}_{\mathrm{n}}}^{\mathrm{dic}}$, and the visual extinction by the torus, $A_{v}$, are related with the magnetic field strength. The magnetic field strength is estimated to be in the range of $12-128 \mathrm{mG}$, for the physical conditions and environments of the gas and dust in the NIR emitting regions of the torus of IC5063.

Key words: AGN, torus-infrared: polarimetry.

\section{Introduction}

The Unified Model (Antonucci, 1993; Urry and Padovani, 1995) of Active Galactic Nuclei (AGN) holds that all AGN are essentially the same object, viewed from different lines of sight (LOS). A geometrically and optically thick, dusty, molecular torus is obscuring the central engine from some LOS. In this scheme, the AGN classification depends solely on the LOS and precise torus properties. In the case when the LOS affords a direct view of the central engine, both the accretion disk and the broad emission line region (BLR) are observed, showing strong UV/optical continuum and broad and narrow emission lines, termed a Type 1 AGN. At larger angles of inclination to our LOS, the torus blocks the radiation from the BLR and continuum with only narrow lines observed, termed a Type 2 AGN.

\footnotetext{
*Augusto Gonzalez Linares Senior Research Fellow
}

Copyright (C) The Society of Geomagnetism and Earth, Planetary and Space Sciences (SGEPSS); The Seismological Society of Japan; The Volcanological Society of Japan; The Geodetic Society of Japan; The Japanese Society for Planetary Sciences; TERRAPUB

doi:10.5047/eps.2013.05.021
The torus absorbs a substantial amount of flux from the central engine and re-radiates this energy in the infrared (IR), predominantly in the mid-infrared (MIR; 7.5-25 $\mu \mathrm{m}$ ). Early models (e.g. Pier and Krolik, 1992; Granato et al., 1997) assumed the material in the torus had a homogeneous distribution. In order to reproduce the observed spectral energy distributions (SED) from wide photometric apertures, these models extend the torus to sizes up to a few hundred parsecs. In the last decade, several studies have constrained the physical boundaries of the torus. The reverberation mapping study by Suganuma et al. (2006) estimated an inner-radii of $0.01-0.1 \mathrm{pc}$, corresponding with the outer radii of the BLR. High-resolution interferometric MIR observations (Tristram et al., 2007) in Circinus suggested a torus outer edge of $2.0 \mathrm{pc}$, with a warm disk-like component of 0.4 pc radius.

Observations and theoretical models assuming homogenous torus have been shown to be in disagreement. Further models assumed a clumpy distribution of the torus (Nenkova et al., 2002, 2008). These models present radiative transfer calculations of a clumpy media, proposing that the dust is distributed in clumps, and correctly accounts for 

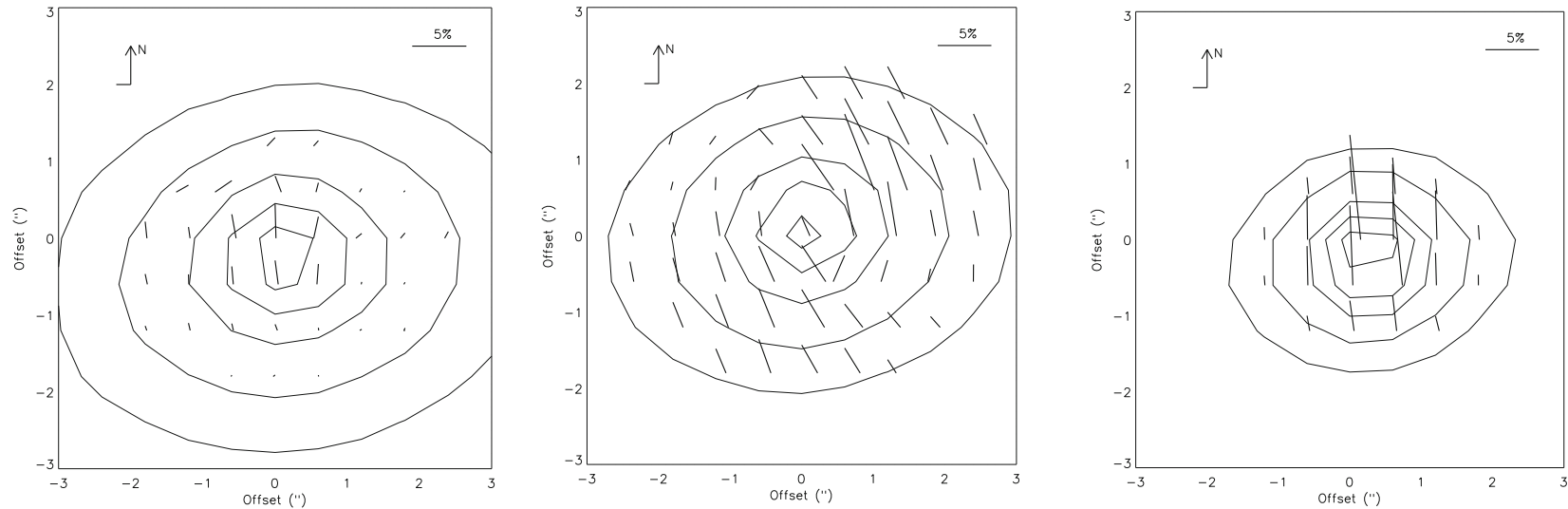

Fig. 1. Total flux contours with polarisation vectors of the central $6^{\prime \prime} \times 6^{\prime \prime}$ at $\mathrm{J}$ (left), $\mathrm{H}$ (middle) and $\mathrm{K}_{\mathrm{n}}$ (right). A vector of $5 \%$ of polarisation is shown at the top right of each plot. The lowest-level total flux contour represents an uncertainty of $0.8 \%$ in the degree of polarisation. Contours are plotted in intervals of $10 \%$ to the peak of the total flux at each band. North is up and East left.
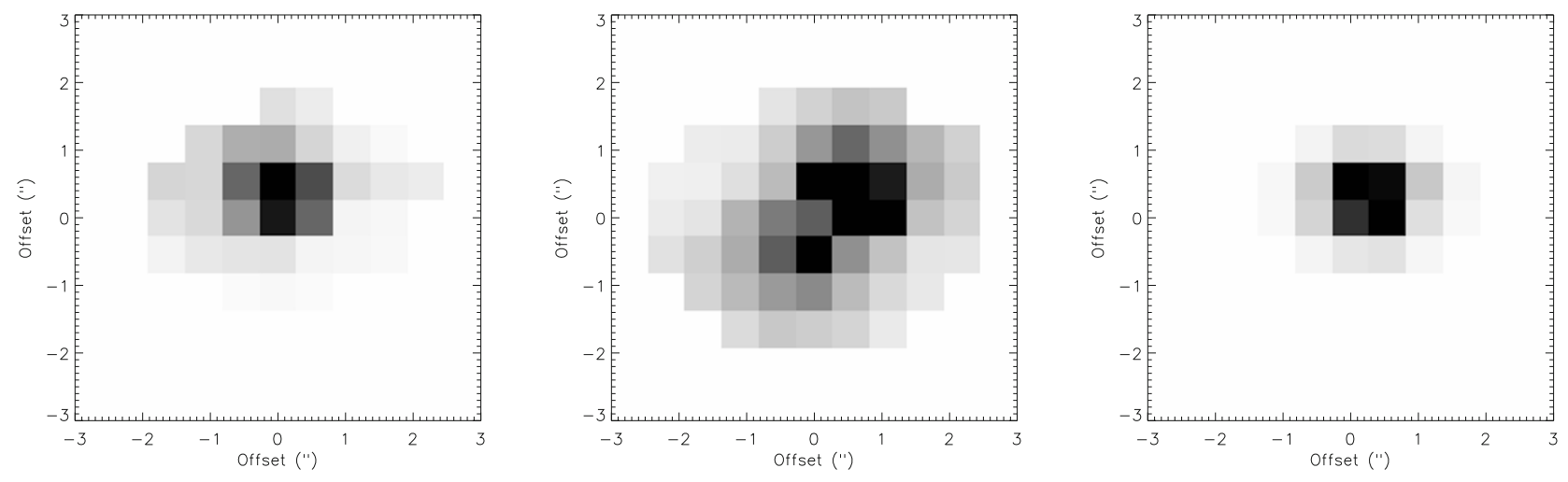

Fig. 2. Polarised flux images of the central $6^{\prime \prime} \times 6^{\prime \prime}$ at $\mathrm{J}($ left $), \mathrm{H}$ (middle) and $\mathrm{K}_{\mathrm{n}}$ (right) bands. North is up and East is left.

(a) the low temperatures close to the central engine; and (b) the variety of SEDs produced by the geometry, clumpy distribution and spectral features, such as the $10 \mu \mathrm{m}$ absorption. The clumpy torus model is able to distribute the clumps in a few parsecs, consistent with the observations (e.g. Radomski et al., 2002; Jaffe et al., 2004; Packham et al., 2005a). This means that the torus cannot be resolved at optical/IR wavelengths, even with the resolution performed from 8-m class telescopes. Recent high-spatial resolution observations of Seyfert galaxies, for both types of AGN, have shown that the clumpy torus model can account for the NIR and MIR emission (see Mason et al., 2006; Ramos Almeida et al., 2009, 2011; Alonso-Herrero et al., 2011). These investigations permit, from a statistical view, an examination of the general properties of the torus, i.e. inclination to our LOS, number of clouds, covering factor, optical depth of clouds, etc. However, the intrinsic properties of the clumpy torus, i.e. the dust grain composition, grain size, grain alignment, etc. remain unknown.

Polarimetry techniques shown a powerful potential to obtain the intrinsic properties of the torus. Several polarimetric studies in the IR to NGC1068 (Mason et al., 2007; Packham et al., 2007) have demonstrated this potential, constraining (1) interstellar dust properties; (2) upper-limit diameter of the torus; (3) mechanisms of polarisation and hence magnetic field directions involved in the central few pc.

With the aim to calculate the magnetic field strength in the torus of IC5063, we used polarimetric observations through the $\mathrm{J}, \mathrm{H}$ and $\mathrm{K}_{\mathrm{n}}$ bands. We developed a polarisation model to fit the total and polarised flux, allow us to interpret the different mechanisms of polarisation to the central engine of IC5063. Further estimates of the extinction to the central engine through different wavelengths, allow us to interpret the origin of the NIR polarisation.

\section{Nuclear Polarisation}

Polarisation maps of the total flux at $J, H$ and $K_{n}$ are shown in Fig. 1. In these figures, the overlaid polarisation vectors are proportional in length to the degree of polarisation with their orientation showing the PA of polarisation. The lowest-level total flux contour represents an uncertainty of $0.8 \%$ in the degree of polarisation. The polarised flux images at $\mathrm{J}, \mathrm{H}$ and $\mathrm{K}_{\mathrm{n}}$ are shown in Fig. 2.

At $\mathrm{J}$, the extended polarisation can be interpreted to be dichroic absorption of diffuse stellar emission though the dust in the nuclear bulge. At $\mathrm{H}$, biconical structure in polarisation flux and the centrosymmetric polarisation pattern in the polarisation maps are observed. This structure has a strongly correspondence with the [OIII] ionised structure observed by Colina et al. (1991) and with the triple structure observed at $8 \mathrm{GHz}$ by Morganti et al. (1998). This spa- 

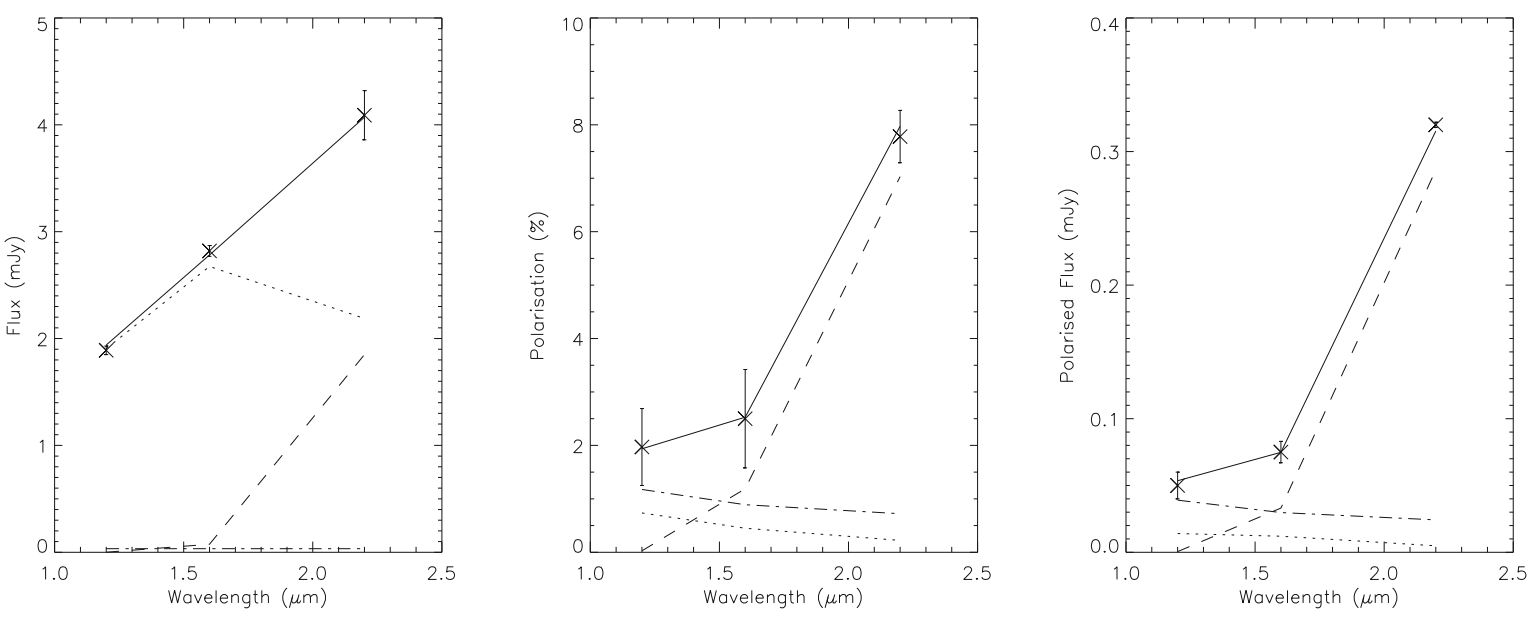

Fig. 3. Model fit to the total flux in a 1.2" aperture (left panel). The diffuse stellar emission through the nuclear bulge (dotted line), AGN through torus (dashed line), ionisation cones (dashed dotted line) and the total (solid line) are shown. Model fit to the degree of polarisation (middle panel) and polarised flux (right panel) in a 1.2" aperture are shown. Polarisation produced through the dichroic absorption by the diffuse stellar emission in the nuclear bulge (dotted line) and within the torus (dashed line), electron scattering (dashed dotted line) and total (solid line) are shown.

tial correspondence provides the first polarised flux imaging observations of the biconical structure of IC5063, consistent with the unified models. At $\mathrm{K}_{\mathrm{n}}$, a highly polarised point-like source is observed.

The degree of polarisation in a $1.2^{\prime \prime}$ aperture at $\mathrm{J}$, $\mathrm{H}$ and $\mathrm{K}_{\mathrm{n}}$ are measured to be $2.7 \pm 0.7 \%, 2.5 \pm 0.9 \%$ and $7.8 \pm 0.5 \%$, respectively. The PA of polarisation is wavelength-independent and measured to be $3 \pm 6^{\circ}$ in the three filters. The measured PA of polarisation is entirely consistent with the PA of polarisation of $3 \pm 2^{\circ}$ measured by Hough et al. (1987) along the J, H and K filters, and $\sim 3^{\circ}$ using optical $(0.45-0.70 \mu \mathrm{m})$ spectropolarimetry by Inglis $e t$ al. (1993).

\section{Polarisation Model}

We have developed a polarisation model to investigate the polarisation mechanisms in the nuclear $1.2^{\prime \prime}$ aperture of IC5063. The near-infrared (NIR) polarisation from IC5063 contains contributions from various processes: (1) dichroic absorption from diffuse stellar emission through dust in the nuclear bulge; (2) electron scattering from ionisation cones; and (3) dichroic absorption arising from aligned dust grains within the torus. The best fit to the measured total and polarised flux, and degree and PA of polarisation are shown in Fig. 3. The model is consistent with dichroic absorption from diffuse stellar emission through dust in the nuclear bulge and electron scattering as dominant mechanisms of polarisation at $\mathbf{J}$ and $\mathrm{H}$. Dichroic absorption from aligned dust grains within the torus of IC5063 is dominant (89\%) at $K_{n}$ with a visual extinction of $A_{v}=48 \pm 2$ mag by the torus.

\section{Intrinsic Polarisation Arising from Dichroic Absorption within the Torus}

The intrinsic polarisation to the central engine of IC5063 arising from dichroic absorption within the torus is estimated. We corrected the measured degree of polarisation of $\mathrm{P}_{\mathrm{K}_{\mathrm{n}}}^{\mathrm{obs}}=7.8 \pm 0.5 \%$ in a $1.2^{\prime \prime}$ aperture at $\mathrm{K}_{\mathrm{n}}$ by (1) the degree of polarisation through dichroic absorption from diffuse stellar emission in an off-nuclear region $\left(\mathrm{P}_{\mathrm{K}_{\mathrm{n}}}^{\text {off }}=0.2 \%\right)$; (2)
Table 1.

\begin{tabular}{lll}
\hline Description & Parameter & Value \\
\hline Gas temperature & $T_{\text {gas }}$ & $10^{4} \mathrm{~K}$ \\
Grain temperature & $T_{\text {gr }}$ & $800-1500 \mathrm{~K}$ \\
Grain size & $a$ & $10^{-6}-10^{-5} \mathrm{~cm}$ \\
Cloud column density & $n$ & $10^{4}-10^{5} \mathrm{~cm}^{-3}$ \\
\hline
\end{tabular}

the estimated polarisation through dichroic absorption from diffuse stellar emission in the nuclear bulge $\mathrm{P}_{\mathrm{K}_{\mathrm{n}}}^{\text {diff }}=0.5-$ $2.7 \%$; (3) the fractional contribution of the AGN to the total flux $\left(\mathrm{AGN}_{\mathrm{K}_{\mathrm{n}}}^{\text {ratio }}=\right.$ Flux $\left._{\mathrm{K}_{\mathrm{n}}}^{\text {total }} / \mathrm{Flux}_{\mathrm{K}_{\mathrm{n}}}^{\mathrm{AGN}}=1.3\right)$; and (4) the fractional contribution of the dichroic absorption within the torus $\left(\mathrm{AGN}_{\mathrm{K}_{\mathrm{n}}}^{\mathrm{dic}}=89 \%\right)$. Specifically, the intrinsic polarisation through dichroic absorption within the torus, $\mathrm{P}_{\mathrm{K}_{n}}^{\mathrm{dic}}$, is estimated as:

$\mathrm{P}_{\mathrm{K}_{\mathrm{n}}}^{\mathrm{dic}}=\left(\mathrm{P}_{\mathrm{K}_{\mathrm{n}}}^{\text {obs }}-\mathrm{P}_{\mathrm{K}_{\mathrm{n}}}^{\mathrm{dic}}-\mathrm{P}_{\mathrm{K}_{\mathrm{n}}}^{\text {diff }}\right) \times\left(1+\mathrm{AGN}_{\mathrm{K}_{\mathrm{n}}}^{\mathrm{ratio}}\right) \times \mathrm{AGN}_{\mathrm{K}_{\mathrm{n}}}^{\mathrm{dic}}$

Finally, the intrinsic polarisation arising from dichroic absorption within the torus is estimated to be $\mathrm{P}_{\mathrm{K}_{\mathrm{n}}}^{\mathrm{dic}}=$ $12.5 \pm 2.7 \%$.

\section{Magnetic Field in the Torus of IC5063}

We considering the physical conditions and environments of the gas and dust within the torus of IC5063. The gas temperature in the BLR reaches values of $T_{\text {gas }}=10^{4} \mathrm{~K}$ (Netzer, 1987). The grain temperature in the clouds of the torus are assumed in the range of 800-1500 K, dependent on the size and grain type i.e. graphite and/or astronomicalsilicates (Nenkova et al., 2008). The grain size is assumed to be in the range of $0.001-0.01 \mu \mathrm{m}$. The column densities of individual clouds was calculated specifically for IC5063, assuming the following parameters: (1) from clumpy torus model, Alonso-Herrero et al. (2011) obtained a radius of $2.4 \mathrm{pc}$ and a number of clouds of $14 \pm 1$; and (2) the gas column densities, as derived from NIR molecular hydrogen lines, ranging 1-10 × $10^{23} \mathrm{~cm}^{-2}$ (Davies et al., 2006; Hicks et al., 2009). The column density for individual clouds in 


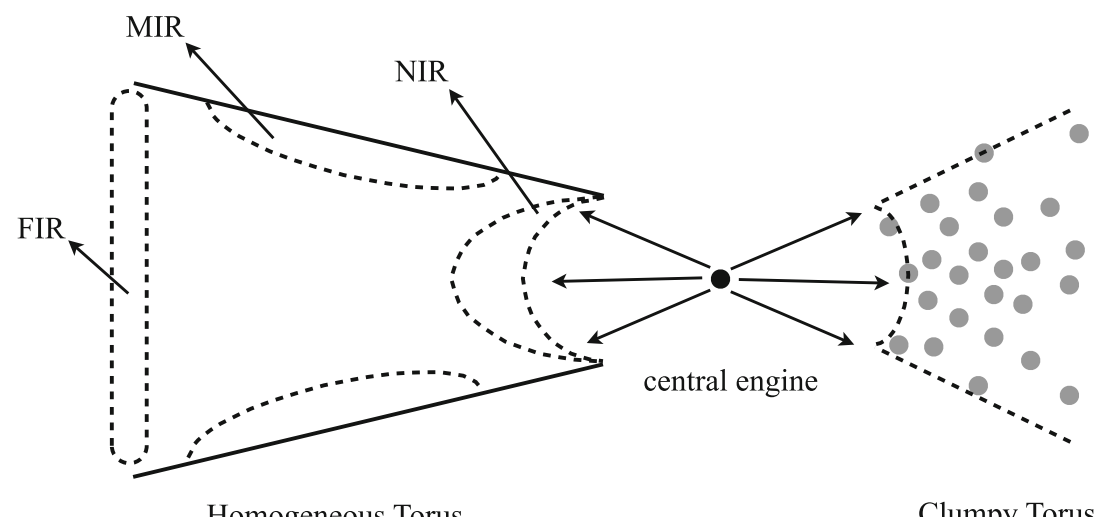

Fig. 4. Sketch of the homogeneous (left) and clumpy torus (right). For the homogeneous torus, the inner side of the torus emits in NIR, the middle regions emits in MIR, and the outer side emits in FIR. For the clumpy torus, the NIR emission arises from the inner side of the directly illuminated clouds, while the MIR and FIR emission is produced from the shadowed side of the clumps (see Fig. 5). Note that clumps located at different distances can produce the same emission at a specific wavelength.

the torus of IC5063 is in the range of $10^{4}-10^{5} \mathrm{~cm}^{-3}$. A summary of these physical parameters is made in Table 1.

Dichroic absorption by aligned dust grains have been attributed to be the polarising mechanism within the torus. Davis and Greenstein (1951) argued that the alignment can be produced by the rotational dynamics of the grain with the environment temperature and/or by the local magnetic field. The orientation of dust grains by a magnetic field is called paramagnetic alignment, through which grains are become oriented with their long axis perpendicular to the magnetic field lines. These models have been highly successfully applied to studies of dust grains in molecular clouds (i.e. Lazarian, 1995; Gerakines et al., 1995). Hence, if we assume that the dust grains within the torus are aligned by paramagnetic relaxation, the polarisation, $\mathrm{P}(\%)$, and the visual extinction, $A_{v}$, are a function of the magnetic field strength (Vrba et al., 1981). Here, an adapted version of equation (8) by Vrba et al. (1981) is presented to be:

$\mathrm{P}(\%) / \mathrm{A}_{\mathrm{v}}=\frac{67 \chi^{\prime \prime} B^{2}}{75 a \omega n}\left(\frac{2 \pi}{m_{H} k T_{\mathrm{gas}}}\right)^{1 / 2}(\gamma-1)\left(1-\frac{T_{\mathrm{gr}}}{T_{\mathrm{gas}}}\right)$

where, $\chi^{\prime \prime}$, is the imaginary part of the complex electric susceptibility, a measure of the attenuation of the wave caused by both absorption and scattering; $B$ is the magnetic field strength; $\gamma$ is the ratio of inertia momentum of the dust grains; $T_{\mathrm{gr}}$, is the grain temperature; $a$, is the grain size; $\omega$, is the orbital frequency of the grains; $n$, is the column density in the cloud; $m_{H}$, is the mass of a hydrogen atom; $k$, is the Boltzmann constant; and $T_{\text {gas }}$, is the gas temperature.

Purcell (1969) showed that the lower bound for the most interstellar grains, the ratio $\chi^{\prime \prime} / \omega$ is (see review Aannestad and Purcell, 1973)

$$
\frac{\chi^{\prime \prime}}{\omega}=2 \times 10^{-12} T_{\mathrm{gr}}^{-1}
$$

The ratio of the inertia momentum of the dust grains, $\gamma$, is defined as:

$$
\gamma=\frac{1}{2}\left[\left(\frac{b}{a}\right)^{2}+1\right]
$$

where, $\frac{b}{a}$, is the grain axial ratio. A typical value of $b / a$ for interstellar dust grains is $\sim 0.2$ (Aannestad and Purcell, 1973; Kim and Martin, 1995).

Although, Vrba et al. (1981) developed the model for Vband, further studies (Gerakines et al., 1995) have shown that is also applicable at $\mathrm{K}$ band. Using the physical conditions shown in Table 1, the intrinsic polarisation arising from dichroic absorption, $\mathrm{P}_{\mathrm{K}_{\mathrm{n}}}^{\mathrm{dic}}=12.5 \pm 2.7 \%$, and the visual extinction, $A_{v}=48 \pm 2 \mathrm{mag}$, by the torus, the magnetic field strength is estimated to be in the range of $12-128 \mathrm{mG}$.

Modjaz et al. (2005) estimated a magnetic field strength of $90 \mathrm{mG}$ for the water vapor maser at $0.2 \mathrm{pc}$ of the central engine of NGC4258 using polarimetric observations with VLA and the GBT at 22GHz. Although for different objects, and at different spatial locations, the range of magnetic field strength we determinate compared well to that by Modjaz et al. (2005).

\section{Discussion}

We present $\mathrm{J}, \mathrm{H}$ and $\mathrm{K}_{\mathrm{n}}$ polarimetry observations of IC5063. The observations shown a highly polarised, $\mathrm{P}_{\mathrm{K}_{\mathrm{n}}}^{\text {obs }}=7.8 \pm 0.5 \%$, point-source at $\mathrm{K}_{\mathrm{n}}$ with a wavelengthindependent PA of polarisation of $3 \pm 6^{\circ}$ in all three filters. To investigate the mechanisms of polarisation to the central engine of IC5063, we developed a polarisation model to fit simultaneously the total and polarised flux, the degree and PA of polarisation in a $1.2^{\prime \prime}(\sim 263 \mathrm{pc})$ aperture of IC5063. The model is consistent with dichroic absorption from diffuse stellar emission through dust in the nuclear bulge and electron scattering as dominant mechanisms of polarisation at $\mathrm{J}$ and $\mathrm{H}$. Dichroic absorption from aligned dust grains within the torus of IC5063 is dominant (89\%) at $K_{n}$ with a visual extinction of $A_{v}=48 \pm 2 \mathrm{mag}$. by the torus. Through the use of various components to the central engine of IC5063, we estimated the intrinsic polarisation from dichroic absorption to be $\mathrm{P}_{\mathrm{K}_{\mathrm{n}}}^{\mathrm{dic}}=12.5 \pm 2.7 \%$.

Estimates of the extinction to the central engine of IC5063 at different wavelengths (X-ray, NIR and MIR) provide further constrains on the extinction by torus. The extinction calculated at those wavelengths, shown wide variations in the visual extinction depending on the wavelengths 


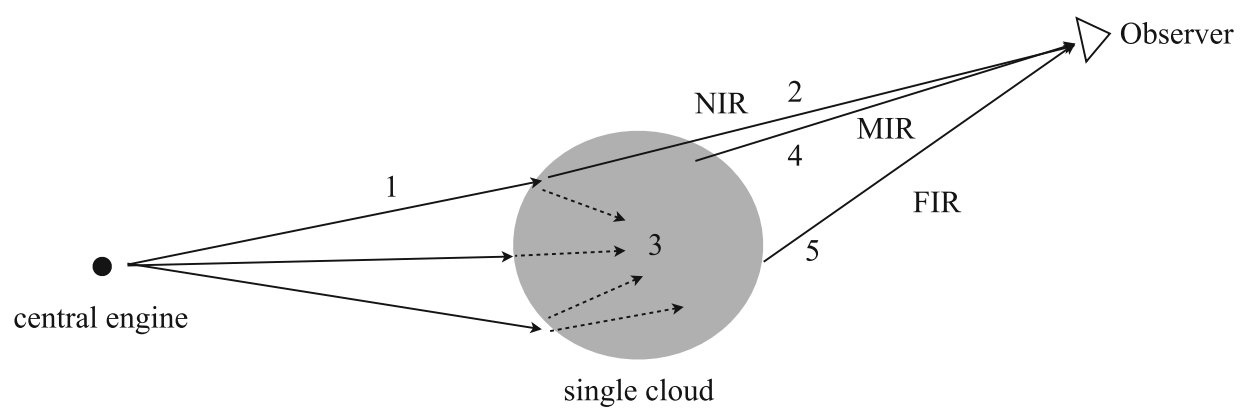

Fig. 5. Sketch of the emission and polarisation of an individual directly illuminated cloud in the clumpy torus. (1) The central engine emits radiation that is absorbed by the cloud, (2) the radiation in the outer layers of the cloud is polarised by the passage of light through the aligned dust grains, and the NIR polarised emission can be in the direction of our LOS. (3) Those rays with direction to the nucleus of the cloud, are completely extinguished. (4) and (5) the difference of temperature in the cloud produces that the warm dust in the middle and back side of the cloud emit in MIR and FIR, respectively.

on which the estimate is based on. Our interpretation of this are (1) the total flux in the NIR wavelengths is from the directly illuminated torus inner-edge or inner-facing dust clump face (i.e. the surface of the dust clump that is directly illuminated by the central engine, Fig. 4) or perhaps the central engine itself (Kishimoto et al., 2005, 2007, 2008); and (2) the NIR polarisation arises as the torus dust grains are aligned, most likely by the central engine's magnetic field (see Fig. 5).

In this scheme, an individual cloud of the clumpy torus can (dependent on the cloud's position and distribution of other clouds) absorb radiation from the central engine on the inner-facing face. This dust will re-emit radiation at NIR wavelengths in all directions. Some of this radiation will be self-absorbed by that clump, and some will be emitted into free space. A small amount of flux will be emitted and penetrate the dust clump at a glancing angle, leading to obscuration and dichroic polarisation from only a portion of the dust clump (see Fig. 5).

We adapted the model by Vrba et al. (1981) to obtain the magnetic field strength in the NIR emotion regions of the torus of IC5063. Note that the model does not take into account for inhomogeneities and/or turbulences within the torus. Hence, our estimate of the magnetic field strength in the range of $12-128 \mathrm{mG}$ represents a lower-limit for the NIR emitting regions of the torus of IC5063.

In this proceedings we are attempting to calculate the magnetic field strength of the clumpy torus in AGN. Specifically, through NIR polarimetry we are attempting to characterize the magnetic field structure below the spatial resolution of the telescope. Hence, wavelength coverage is crucial in our study. High-spatial resolution plays an important role in the refinement and/or modification of the models presented here. The next generation of polarimeters, such as adaptive optics optimized imaging polarimeter in the NIR (1-5 $\mu \mathrm{m})$ MMT-POL (Packham et al., 2010) at the 6.5-m MMT, the MIR polarimeter $(7.5-13 \mu \mathrm{m}) \mathrm{Ca}-$ nariCam (Packham et al., 2005b) at the 10.4-m GTC will provide a high spatial resolution and polarisation sensibility that will allow us to refine and/or modify these studies. Also, mm-polarimetric observations with ALMA will allow to refine intrinsic properties of the torus, i.e. dust density, grain sizes, temperature.
Acknowledgments. E. Lopez-Rodriguez acknowledges UF Alumni Fellowship 2008-2012. C. Ramos Almeida acknowledges financial support from the Spanish Ministry of Science and Innovation (MICINN) through project Consolider-Ingenio 2010 Program grant CSD2006-00070: First Science with the GTC (http://www.iac.es/consolider-ingenio-gtc/) and the Estallidos group through project PN AYA2010-21887-C04.04. A. AlonsoHerrero acknowledges support from the Spanish Plan Nacional de Astronomía y Astrofísica under grant AYA2009-05705-E.

\section{References}

Aannestad, P. A. and E. M. Purcell, Interstellar grains, Ann. Rev. Astron. Astrophys., 11, 309-362, 1973.

Alonso-Herrero, A., C. Ramos-Almeida, R. Mason, A. Asensio Ramos, P. F. Roche, N. A. Levenson, M. Elitzur, J. M. Rodriguez Espinosa, S. Young, T. Diaz-Santos, and A. M. Perez Garcia, Torus and active galactic nucleus properties of nearby seyfert galaxies: Results from fitting infrared spectral energy distributions and spectroscopy, Astrophys. J., 736, 82-112, 2011.

Antonucci, R., Unified models for active galactic nuclei and quasars, Ann. Rev. Astron. Astrophys., 31, 473-521, 1993.

Colina, L., W. B. Sparks, and F. Macchetto, IC 5063-A merger remnant with a hidden luminous active nucleus, Astrophys. J., 370, 102-117, 1991.

Davies, R. I., J. Thomas, R. Genzel, F. Mueller Sanchez, L. J. Tacconi, A. Sternberg, F. Eisenhauer, R. Abuter, R. Saglia, and R. Bender, The star-forming torus and stellar dynamical black hole mass in the seyfert 1 nucleus of NGC3227, Astrophys. J., 646, 754-773, 2006.

Davis, L. J. and J. L. Greenstein, The polarization of starlight by aligned dust grains, Astrophys. J., 114, 206-240, 1951.

Gerakines, P. A., D. C. B. Whittet, and A. Lazarian, Grain alignment in the Taurus Dark Cloud, Astrophys. J., 445, 171-175, 1995.

Granato, G. L., L. Danese, and A. Franceschini, Thick Tori around active galactic nuclei: The case for extended Tori and consequences for their X-ray and infrared emission, Astrophys. J., 486, 147-159, 1997.

Hicks, E. K. S., R. I. Davies, M. A. Malkam, R. Genzel, L. J. Tacconi, F. Muller Sanchez, and A. Sternberg, The role of molecular gas in obscuring seyfert active galactic nuclei, Astrophys. J., 696, 448-470, 2009.

Hough, J. H., C. Brindle, D. J. Axon, J. Bailey, and W. B. Sparks, Infrared and optical polarimetry of the radio elliptical IC 5063 (PKS2048-57)Discovery of a highly polarized non-thermal nucleus, Mon. Not. R. Astron. Soc., 224, 1013-1018, 1987.

Inglis, M. D., C. Brindle, J. H. Hough, S. Young, D. J. Axon, J. A. Bailey, and M. J. Ward, Evidence for an obscured broadline region in the early type radio galaxy IC5063, Mon. Not. R. Astron. Soc., 263, 895-902, 1993.

Jaffe, W., K. Meisenheimer, H. J. A. Rottgering, Ch. Leinert, A. Richichi, O. Chesneau, D. Fraix-Burnet, A. Glazenborg-Kluttig, G.-L. Granato, U. Graser, B. Heijligers, R. Kohler, F. Malbet, G. K. Miley, F. Paresce, J.-W. Pel, G. Perrin, F. Przygodda, M. Schoeller, H. Sol, L. B. F. M. Waters, G. Weigelt, J. Woillez, and P. T. de Zeeuw, The central dusty torus in the active nucleus of NGC 1068, Nature, 6987, 47-49, 2004. 
Kim, S. H. and P. G. Martin, The size distribution of interstellar dust particles as determined from polarization: Spheroids, Astrophys. J., 444, 293-305, 1995.

Kishimoto, M., R. Antonucci, and O. Blaes, The dust-eliminated shape of quasar spectra in the near-infrared: a hidden part of the big blue bump, Mon. Not. R. Astron. Soc., 364, 640-648, 2005.

Kishimoto, M., S. F. Honig, T. Beckert, and G. Weigelt, The innermost region of AGN tori: implications from the HST/NICMOS type 1 point sources and near-IR reverberation, Astron. Astrophys., 476, 713-721, 2007.

Kishimoto, M., R. Antonucci, O. Blaes, A. Lawrence, C. Boisson, M. Albrecht, and C. Leipski, The characteristic blue spectra of accretion disks in quasars as uncovered in the infrared, Nature, 454, 492-494, 2008 .

Lazarian, A., Davis-Greenstein alignment of nonspherical grains, Astrophys. J., 453, 229-237, 1995.

Mason, R. E., T. R. Geballe, C. Packham, N. A. Levenson, M. Elitzur, R. S. Fisher, and E. Perlman, Spatially resolved mid-infrared spectroscopy of NGC 1068: The nature and distribution of the nuclear material, Astrophys. J., 640, 612-624, 2006.

Mason, R. E., G. S. Wright, A. Adamson, and Y. Pendleton, Spectropolarimetry of the $3.4 \mu \mathrm{m}$ absorption feature in NGC 1068, Astrophys. J., 656, 798-804, 2007.

Modjaz, M., J. M. Moran, P. T. Kondratko, and Li. J. Greenhill, Probing the magnetic field at subparsec radii in the accretion disk of NGC 4258, Astrophys. J., 626, 104-119, 2005.

Morganti, R., T. Oosterloo, and Z. Tsvetanov, A radio study of the Seyfert Galaxy IC 5063: Evidence for fast gas outflow, Astron.J., 115, 915-927, 1998.

Nenkova, M., Z. Ivezic, and M. Elitzur, Dust emission from active galactic nuclei, Astrophys. J., 570, L9-L12, 2002.

Nenkova, M., M. M. Sirocky, Z. Ivezic, and M. Elitzur, AGN dusty tory I. Handling of clumpy media, Astrophys. J., 685, 147-159, 2008.

Netzer, H., Quasar disk-II. A composite model for the broad-line region, Mon. Not. R. Astron. Soc., 225, 55-72, 1987.

Packham, C., J. T. Radomski, P. F. Roche, D. K. Aitken, E. Perlman, A. Alonso-Herrero, L. Colina, and C. M. Telesco, The extended midinfrared structure of the circinus galaxy, Astrophys. J., 618, L17-L20, 2005a

Packham, C., J. H. Hough, and C. M. Telesco, CanariCam-polarimetry: A dual-beam $10 \mu \mathrm{m}$ polarimeter for the GTC, ASPC, 343, 38-42, 2005b.

Packham, C., S. Young, S. Fisher, K. Volk, R. Mason, J. H. Hough, P.
F. Roche, M. Elitzur, J. Radomski, and E. Perlman, Gemini Mid-IR polarimetry of NGC 1068: Polarized structures around the nucleus, Astrophys. J., 661, L29-L32, 2007.

Packham, C., T. J. Jones, M. Krejny, K. Dewahl, C. Warner, and E. Lopez Rodriguez, Progress on MMT-POL: the 1-5 $\mu \mathrm{m}$ adaptive optics optimized polarimeter for the MMT, SPIE, 7735, 77356J-77356J-10, 2010

Pier, E. A. and J. H. Krolik, Radiation-pressure-supported obscuring tori around active galactic nuclei, Astrophys. J., 399, L23-L26, 1992.

Purcell, E. M., On the absorption and emission of light by interstellar grains, Astrophys. J., 159, 433-440, 1969.

Radomski, J. T., R. K. Piña, C. Packham, C. M. Telesco, and C. N. Tadhunter, High-resolution mid-infrared morphology of Cygnus A, Astrophys. J., 566, 675-681, 2002.

Ramos Almeida, C., N. A. Levenson, J. M. Rodriguez Espinosa, A Alonso-Herrero, A. Asensio Ramos, J. T. Radomski, C. Packham, R. S. Fisher, and C. M. Telesco, The infrared nuclear emission of Seyfert Galaxies on parsec scales: Testing the clumpy torus models, Astrophys. J., 702, 1127-1149, 2009.

Ramos Almeida, C., N. A. Levenson, A. Alonso-Herrero, A. Asensio Ramos, J. M. Rodriguez Espinosa, A. M. Perez Garcia, C. Packham, R. Mason, J. T. Radomski, and T. Diaz-Santos, Testing the unification model for active galactic nuclei in the infrared: Are the obscuring Tori of Type 1 and 2 Seyferts different?, Astrophys. J., 731, 92-104, 2011.

Suganuma, M., Y. Yoshii, Y. Kobayashi, T. Minezaki, K. Enya, H. Tomita T. Aoki, S. Koshida, and B. A. Peterson, Reverberation measurements of the inner radius of the dust Torus in nearby Seyfert 1 Galaxies, Astrophys. J., 639, 46-63, 2006.

Tristram, K. R. W., K. Meisenheimer, W. Jaffe, M. Schartmann, H. W. Rix, Ch. Leinert, S. Morel, M. Wittkowski, H. Röttgering, G. Perrin, B. Lopez, D. Raban, W. D. Cotton, U. Graser, F. Paresce, and Th. Henning, Resolving the complex structure of the dust torus in the active nucleus of the Circinus galaxy, Astron. Astrophys., 474, 837-850, 2007.

Urry, C. M. and P. Padovani, Unified schemes for radio-loud active galactic nuclei, PASP, 107, 803-845, 1995.

Vrba, F. J., G. V. Coyne, and S. Tapia, Observations of grain and magnetic field properties of the R Coronae Australis dark cloud, Astrophys. J., 243, 489-511, 1981.

E. Lopez-Rodriguez (e-mail: elr@astro.ufl.edu), C. Packham, S. Young, M. Elitzur, N. A. Levenson, R. E. Mason, C. Ramos Almeida, A. Alonso-Herrero, T. J. Jones, and E. Perlman 